\title{
ON THE EXISTENCE OF SOLUTIONS TO A SPECIAL VARIATIONAL PROBLEM
}

\author{
ARRIGO CELLINA AND MIHAI VORNICESCU
}

\begin{abstract}
In this paper we establish an existence and regularity result for solutions to the problem

$$
\operatorname{minimize} \int_{\Omega} L(|\nabla u(x)|) d x \quad \text { on }\left\{u: u-u_{0} \in W_{0}^{1,1}(\Omega)\right\}
$$

for boundary data that are constant on each connected component of the boundary of $\Omega$. The Lagrangean $L$ belongs to a class that contains both extended valued Lagrangeans and Lagrangeans with linear growth. Regularity means that the sulution $u$ is Lipschitz continuous and that, in addition, $\left\|L^{\prime}(|\nabla \tilde{w}|)\right\|_{\infty}$ is bounded.
\end{abstract}

\section{INTRODUCTION}

In the present paper, $\Omega$ is a bounded open subset of $\mathbb{R}^{N}$, with a $C^{2}$ boundary $\partial \Omega$, whose connected components will be denoted by $\Gamma_{i}$. We consider the special problem of minimizing

$$
\int_{\Omega} L(|\nabla u(x)|) d x
$$

on $u \in \phi+W_{0}^{1,1}(\Omega)$, where we assume that the restriction of the boundary data $\phi$ to each $\Gamma_{i}$ is a constant, $k_{i}$. The simplest example of this situation is provided by an annulus in $\mathbb{R}^{2}$, when $\phi$ is constant on each of the two radii. The minimal surface problem offers an example of a minimization problem of this type where the solution to the minimum problem either does not exist or it exists but fails to be Lipschitz continuous: in fact, the special nature of this problem, in itself, prevents the validity of the mean curvature condition, which is a sufficient condition to ensure the existence of solutions in the case of minimal surfaces [3], [4].

Our purpose here is to prove existence and regularity of solutions. Existence is meant in $\phi+W_{0}^{1,1}(\Omega)$. By regularity, we mean more than Lipschitz continuity: for the class of problems we wish to discuss, where $L$ can be extended valued, the Lipschitz continuity of the solution, by itself, would not be relevant enough. In fact, for instance, consider the case where the Lagrangean $L$ is $L(t)=\frac{1}{4} \frac{1}{1-|t|}$ for $|t|<1,=+\infty$ elsewhere: then, every $w$ that makes the integral functional finite, independent on whether it is a solution or not, has to be Lipschitz continuous. A possible notion of regularity for the case of extended-valued Lagrangeans would be to require both that $\nabla \tilde{w} \in L^{\infty}$ and $L^{\prime}(|\nabla \tilde{w}|) \in L^{1}$. In this paper we seek, and we prove, more: we will call regular a map $w$ when $\|\nabla \tilde{w}\|_{L^{\infty}}$ and $\left\|L^{\prime}(|\nabla \tilde{w}|)\right\|_{L^{\infty}}$ are both bounded. Our Theorem 1 provides existence of solutions for a broad class of

1991 Mathematics Subject Classification. 49K20.

Key words and phrases. Existence of solutions, growth conditions. 
Lagrangeans, containing Lagrangeans of linear growth and Lagrangeans that are extended valued: in fact, no specific growth assumption appears: $L$ has to belong to a class of convex functions, invariant by polarity, defined through the requirement of continuity of the function itself and of its polar.

\section{MAin RESUlts}

$\overline{\mathbb{R}}$ is $\mathbb{R} \cup+\infty$. We denote by $\operatorname{Dom}(L)$ the effective domain of $L$. By $L^{*}$ we mean the polar or Legendre transform of $L$, a (possibly extended valued) convex function $[6]$.

By $\pi(x)$ we denote the subset of $\partial \Omega$ of points nearest to $x \in \Omega$, i.e., $\pi(x)=\{y \in$ $\partial \Omega:|x-y|=\operatorname{dist}(x, \partial \Omega)\}$. We shall consider points $x$ sufficiently close to $\partial \Omega$ to have an unique projection on it. Set $c_{i}(y)$ be the curvatures of $\partial \Omega$ at $y$, define $r_{i}$ by $c_{i}(y)=-\frac{1}{r_{i}(y)}$ : from the smoothness of $\partial \Omega$ we have that

$$
r^{*}=\inf _{i} \inf _{y}\left|r_{i}(y)\right|>0 .
$$

It is well known, [2], [1], that $\Delta d(x)=\sum_{1}^{N-1} \frac{1}{r_{i}(\pi(x))+d(x)}$.

In what follows, $\operatorname{Lip}_{0}(\Omega)$ is the linear space of Lipschitz continuous functions vanishing at the boundary of $\Omega$. We shall consider the problem of minimizing (1) on $\phi+\operatorname{Lip}_{0}(\Omega)$ and on $\phi+W_{0}^{1,1}(\Omega)$. A function $w$ is called regular if both $\|\nabla w\|_{\infty}$ and $\left\|L^{\prime}(|\nabla w|)\right\|_{\infty}$ are bounded.

We consider the class $\mathbb{C} \mathbb{C}$ of convex Lagrangeans defined below.

Definition 1. $L: \mathbb{R} \rightarrow \overline{\mathbb{R}}$ is in $\mathbb{C} \mathbb{C}$ if it is a (possibly extended valued) symmetric, convex function, twice differentiable on the interior of its effective domain, and with $L^{\prime \prime}>0$ there, and such that $0 \in \operatorname{int} \operatorname{Dom}(L)$ and $L(0)=L^{\prime}(0)=0$. In addition, both $L$ and $L^{*}$ are continuous.

The assumption $L(0)=0$ is satisfied by any convex function, possibly by adding a constant, that has no effect on the minimization problem; the assumption $L^{\prime}(0)=0$ is needed to have differentiability at $0 ; L^{\prime \prime}>0$ assures the uniqueness of the solution.

The minimal surface functional, where $L(t)=\sqrt{1+t^{2}}-1$, satisfies all the requirements for being in $\mathbb{C} \mathbb{C}$ with the only exception that its polar, i.e. the convex function $L^{*}(p)=-\sqrt{1-p^{2}}+1$ for $|p| \leq 1,=+\infty$ elsewhere, is not continuous (as an extended-valued function.) Hence, Theorem 1, that follows, does not apply to the minimal surface functional, as it has to be: in fact, it is well known that the claim of the theorem is false in this case.

We have the following proposition

Proposition 1. Let $L$ be in $\mathbb{C} \mathbb{C}$. Then $L^{*}$ is in $\mathbb{C} \mathbb{C}$.

Proof. From the definition of polar, it follows that $L^{*}(0)=0$. From $\left(L^{*}\right)^{\prime}\left(L^{\prime}(0)\right)=$ 0 follows that $\left(L^{*}\right)^{\prime}(0)=0$; moreover, for $\hat{p} \operatorname{in} \operatorname{int}\left(\operatorname{Dom}\left(L^{*}\right)\right)$, we have that $\left(L^{*}\right)^{\prime \prime}$ exists and is positive: in fact, fix $\hat{p}$ and set $\hat{\xi}=\left(L^{*}\right)^{\prime}(\hat{p})$. Write

$$
\frac{\left(L^{*}\right)^{\prime}(p)-\left(L^{*}\right)^{\prime}(\hat{p})}{p-\hat{p}}=\frac{\left(L^{*}\right)^{\prime}\left(L^{\prime}(x)\right)-\left(L^{*}\right)^{\prime}\left(L^{\prime}(\hat{x})\right)}{L^{\prime}(x)-L^{\prime}(\hat{x})}=\frac{x-\hat{x}}{L^{\prime}(x)-L^{\prime}(\hat{x})}
$$

to obtain that $\left(L^{*}\right)^{\prime \prime}(\hat{p})=\frac{1}{L^{\prime \prime}\left(\left(L^{*}\right)^{\prime}(\hat{p})\right)}$.

Being $\mathbb{C} \mathbb{C}$ invariant under polarity, does not, in itself, contain any specific growth assumption. 
The following proposition will be of use. Recall that, by assumption, $L^{\prime}(0)=0$ : hence, by the notation $L^{\prime}(|\xi|) \frac{\xi}{|\xi|}$, we mean $L^{\prime}(|\xi|) \frac{\xi}{|\xi|}$, when $\xi \neq 0$, and 0 , when $\xi=0$.

Proposition 2. Let $L$ be in $\mathbb{C} \mathbb{C}$, let $w$, a solution to the problem of minimizing (1) on $\phi+\operatorname{Lip}_{0}(\Omega)$, be regular. Then the Euler Lagrange equation holds, i.e., for every $\eta$ in $W_{0}^{1,1}(\Omega)$, we have

$$
\int_{\Omega} L^{\prime}(|\nabla u(x)|)\left\langle\frac{\nabla u(x)}{|\nabla u(x)|}, \nabla \eta(x)\right\rangle d x=0
$$

Proof. The assumption of regularity implies that, in either case when $\operatorname{Dom}(L)=$ $(-\ell,+\ell)$ or $\operatorname{Dom}(L)=(-\infty,+\infty)$, there exist $K$ and $\delta$ such that $|\nabla u(x)| \leq K$ and $(-K-\delta, K+\delta) \subset \subset \operatorname{Dom}(L)$, so that $L^{\prime}$ is uniformly bounded on $(-K-\delta, K+\delta)$. For fixed $\eta$ in $C_{c}^{\infty}(\Omega)$, for every $\varepsilon$ small, $|\nabla u(x)+\varepsilon \nabla \eta(x)|<K+\delta$, and one can pass to the limit under the integral sign. This establishes the validity of (3) for $\eta$ in $C_{c}^{\infty}(\Omega)$. Moreover, $\nabla L(|\nabla \tilde{w}|) \in L^{\infty}(\Omega)$, so that equation (3) holds also for $\eta \in W_{0}^{1,1}(\Omega)$.

The following is our main result. Recall that $\Gamma_{i}$ are the connected components of $\partial \Omega$.

Theorem 1. Let $L$ be in $\mathbb{C} C$. Let each restriction of the boundary datum $\phi$ to $\Gamma_{i}$ be constant. Then, whenever $\phi$ is regular, a solution $\tilde{w}$ to the problem of minimizing (1) on $u \in \phi+W^{1,1}(\Omega)$, exists, is unique and it is regular.

Notice that, in the case where $L$ takes values in $\mathbb{R}$, then, no matter what the values $k_{i}$ are, the restriction of $\phi$ to $\partial \Omega$ can be extended to $\Omega$ so as to make $\phi$ regular.

The following Lemmas will be used in the Proof. Opposite to Theorem 1, the validity of Lemma 1 does not depend on any special condition on the behaviour of $\phi$ on the boundary of $\Omega$.

Lemma 1 (A Maximum Principle for extended valued Lagrangeans). Let $L: \mathbb{R} \rightarrow$ $\overline{\mathbb{R}}$ be convex and continuous, with $\operatorname{Dom}(L)=(-\ell,+\ell)$, and let it be twice differentiable on its effective domain, with $L^{\prime \prime}>0$ there. Let $\tilde{w}$ be a solution to the problem of minimizing

$$
\int_{\Omega} L(|\nabla u(x)|) d x
$$

on $\phi+W_{0}^{1,1}(\Omega)$, where $\phi$ is Lipschitz continuous of Lipschitz constant $\lambda<\ell$. Then, for no $x^{*} \in \Omega$ there exists $y^{*} \in \pi\left(x^{*}\right)$, such that $\left|\tilde{w}\left(x^{*}\right)-\phi\left(y^{*}\right)\right|=\ell\left|x^{*}-y^{*}\right|$.

Proof. Assume that such $x^{*}$ and $y^{*}$ exist. Let $g=g(t)$ be defined by

$$
L^{\prime}(g(t))=L^{\prime}\left(\frac{\ell}{2}\right) \frac{1}{t^{N-1}} .
$$

The map $g$ is the solution to the differential equation

$$
-\frac{L^{\prime \prime}}{L^{\prime}}(v(r)) \frac{d v}{d r}=\frac{N-1}{r}
$$

satisfying $v(1)=\frac{\ell}{2}$; in particular, $g^{\prime}(t)<0$. We have that $g(t) \rightarrow \ell^{-}$as $t \rightarrow 0^{+}$: hence, fix $\lambda<\Lambda<\ell$ and let $\delta<1$ be such that $t<\delta$ implies $g(t)>\Lambda$. Let $D$ be the diameter of $\Omega$ and choose $\tilde{r}^{0}$ so large that $\frac{D}{\tilde{r}^{0}}<\delta$. 
Set $w(x)=\int_{0}^{\left|x-y^{*}\right|} g\left(\frac{s}{\tilde{r}^{0}}\right) d s+\phi\left(y^{*}\right): w$ is a pointwise solution to

$$
\operatorname{div}_{x} \nabla_{\xi} L(|\nabla v(x)|)=0
$$

such that $w\left(y^{*}\right)=\phi\left(y^{*}\right)$ and such that, on $\left\{\left|x-y^{*}\right|=\tilde{r}^{0}\right\}$, we have $\nabla w(x)=$ $\frac{\ell}{2} \frac{x-y^{*}}{\left|x-y^{*}\right|}$; we claim that it is also a solution to the Euler Lagrange equation, in the sense that, for every $\eta$ Lipschitz continuous with $\eta(y)=0$ when $y \in \partial \Omega$, we have

$$
\int_{\Omega}\left\langle\nabla_{\xi} L(|\nabla w(x)|), \nabla \eta(x)\right\rangle d x=\int_{\Omega} L^{\prime}\left(\frac{\ell}{2}\right) \frac{1}{\left|x-y^{*}\right|^{N-1}}\left\langle\frac{x-y^{*}}{\left|x-y^{*}\right|}, \nabla \eta(x)\right\rangle d x=0 .
$$

Set $E^{+}$be the open set $\{x: \eta(x)>0\}$ and analogously for $E^{-}$. We shall prove that

$$
\int_{E^{+}} \frac{1}{\left|x-y^{*}\right|^{N-1}}\left\langle\frac{x-y^{*}}{\left|x-y^{*}\right|}, \nabla \eta(x)\right\rangle d x \text { and } \int_{E^{+}} \frac{1}{\left|x-y^{*}\right|^{N-1}}\left\langle\frac{x-y^{*}}{\left|x-y^{*}\right|}, \nabla \eta(x)\right\rangle d x
$$

are zero. Consider polar coordinates $(\omega, r)$ centered at $y^{*}$ : the intersection of a half line $L_{c}=\{\omega=c, r \geq 0\}$ with the open set $E^{+}$can be described as $\{\omega=c ; r \in$ $\left.\cup_{i}\left(\alpha_{i}(c), \beta_{i}(c)\right)\right\}$ where some or all of the $\left\{\omega=c ; \alpha_{i}(c)\right\}$ and of the $\left\{\omega=c ; \beta_{i}(c)\right\}$ can belong to $\partial \Omega$. On almost every half line, the derivative of the map $r \rightarrow \eta\left(\omega^{\prime} r\right)$ equals a.e. $\left\langle\omega, \nabla \eta\left(\omega^{\prime} r\right)\right\rangle$ so that

$$
\int_{E^{+}} \frac{1}{\left|x-y^{*}\right|^{N-1}}\left\langle\frac{x-y^{*}}{\left|x-y^{*}\right|}, \nabla \eta(x)\right\rangle d x=\int_{|\omega|=1}\left(\Sigma_{i} \int_{\alpha_{i}(c)}^{\beta_{i}(c)} \frac{c}{r^{N-1}}\left(\frac{d}{d r}(\eta(r \omega))\right) r^{N-1} d r\right) d \omega .
$$

For each $i, \eta\left(\alpha_{i}(c), c\right)=0$ and the same is true at $\left\{\omega=c ; \beta_{i}(c)\right\}$. Hence we obtain that the last integral is zero. Thus we prove the claim, and the convexity of $L$ implies that $w$, a solution to the Euler Lagrange equation, is also a solution to the minimization problem.

Let $y \in \bar{\Omega}$ : then $w(y)-w\left(y^{*}\right)=\int_{0}^{\left|y-y^{*}\right|} g\left(\frac{s}{\tilde{r}^{0}}\right) d s$ and, by our choice of $\tilde{r}^{0}$, $\frac{\left|y-y^{*}\right|}{\tilde{r}^{0}} \leq \frac{D}{\tilde{r}^{0}}<\delta$ and we infer $g\left(\frac{s}{\tilde{r}^{0}}\right)>\Lambda$, so that $w(y)-w\left(y^{*}\right)>\Lambda\left|y^{*}-y\right|$. On the other hand, for $y \in \partial \Omega$, we have $\left|\tilde{w}(y)-\tilde{w}\left(y^{*}\right)\right|=\left|\phi(y)-\phi\left(y^{*}\right)\right| \leq \lambda\left|y^{*}-y\right|$; hence, on $\partial \Omega$, one has $w \geq \tilde{w}$. By assumption, at the point $x^{*}$ we have $\left|\tilde{w}\left(x^{*}\right)-\phi\left(y^{*}\right)\right|=$ $\ell\left|y^{*}-x^{*}\right|$ while $w\left(x^{*}\right)-\phi\left(y^{*}\right)=\int_{0}^{\left|x^{*}-y^{*}\right|} g\left(\frac{s}{\tilde{r}^{0}}\right) d s<\ell\left|y^{*}-x^{*}\right|$. Hence the set $\{x \in \Omega: \tilde{w}(x)>w(x)\}$ is an open set containing $x^{*}$ : on the connected component containing $x^{*}$ there are two solutions with the same boundary data, a contradiction to the assumption of strict convexity of $L$.

Lemma 2. Let $L: \mathbb{R} \rightarrow \mathbb{R}$ be convex and twice differentiable. Then, $\int^{\infty} t L^{\prime \prime}(t) d t=$ $+\infty$ if and only if $\int^{\infty} t \frac{L^{\prime \prime}(t)}{L^{\prime}(t)} d t=+\infty$.

Proof. a): only if.

Since $L^{\prime}$ is increasing, we have

$$
\int_{\alpha}^{\beta} t \frac{L^{\prime \prime}(t)}{L^{\prime}(t)} d t \geq \frac{1}{L^{\prime}(\beta)} \int_{\alpha}^{\beta} t L^{\prime \prime}(t) d t
$$

and both $\lim _{\beta \rightarrow \infty}\left(L^{\prime}(\beta)\right)=+\infty$ and $\lim _{\beta \rightarrow \infty} \int_{\alpha}^{\beta} t L^{\prime \prime}(t) d t=+\infty$. By l'Hopital rule,

$$
\lim _{\beta \rightarrow \infty} \frac{1}{L^{\prime}(\beta)} \int_{\alpha}^{\beta} t L^{\prime \prime}(t) d t=\lim _{\beta \rightarrow \infty} \frac{\beta L^{\prime \prime}(\beta)}{L^{\prime \prime}(\beta)}=+\infty .
$$

b): if.

$L^{\prime}$ is bounded below by a positive constant. 
Proof of Theorem 1. Dom $\left(L^{*}\right)$ is the open interval from $-\ell^{*}$ to $+\ell^{*}$, with $\ell^{*}$ possibly $=\infty$.

The proof will consist in the construction of a suitable barrier. To prove Lipschitz continuity of solutions, affine barriers have been used, as in [5], through the bounded slope condition, as well as barriers of more general form, as in [1], [2], for the minimal surface case. The construction of our barrier will differ from those appearing in the papers quoted above.

We shall first prove the existence of a solution to the minimization problem (1) on $\phi+\operatorname{Lip}_{0}(\Omega)$. Let $d^{*}>0$ be so small that $d^{*}<r^{*}$, where $r^{*}$ is defined in (2) so that for $\operatorname{dist}(x, \partial \Omega) \leq d^{*}$, we have that $\pi(x)$ is single valued; set $d(x)=|x-\pi(x)|$. Fix $i$ : we shall define a barrier on $\left\{x \in \Omega\right.$ : $\left.\operatorname{dist}\left(x, \partial \Omega_{i}\right) \leq d^{*}\right\}$.

When $v(x)=\psi(d(x))$, one obtains:

$$
\operatorname{div}_{x} \nabla_{\xi} L(|\nabla v(x)|)=L^{\prime}\left(\left|\psi^{\prime}(d(x))\right|\right) \operatorname{sign}\left(\psi^{\prime}(d(x))\right) \Delta d(x)+\psi^{\prime \prime}(d(x)) L^{\prime \prime}\left(\left|\psi^{\prime}(d(x))\right|\right)
$$

i) Case $\operatorname{Dom}(L)=(-\infty,+\infty)$.

Let $v^{*}=\sup _{y, z \in \partial \Omega}|\phi(z)-\phi(y)|$ : as it is well known, when $\tilde{w}$ is a solution, for $x \in \Omega$ and each $i,\left|\tilde{w}-k_{i}\right| \leq v^{*}$. Set $D=\sup \left\{|\Delta(x)|: x \in \Omega, d(x) \leq d^{*}\right\}:$ from the assumptions of regularity of $\partial \Omega$ and the choice of $d^{*}, D$ is finite.

Let $\psi$ be a solution to

$$
L^{\prime}\left(\psi^{\prime}(d)\right) D+\psi^{\prime \prime}(d) L^{\prime \prime}\left(\psi^{\prime}(d)=0\right.
$$

For generic $\ell_{1}$ and $\ell_{2}$, we have

$$
\int_{\ell_{1}}^{\ell_{2}} \psi^{\prime}(\ell) d \ell=\int_{\psi^{\prime}\left(\ell_{1}\right)}^{\psi^{\prime}\left(\ell_{2}\right)} \psi^{\prime} \frac{d \ell}{d \psi^{\prime}} d \psi^{\prime}
$$

and, from $\psi^{\prime \prime}\left(\ell\left(\psi^{\prime}\right)=\frac{1}{\ell^{\prime}\left(\psi^{\prime}\right)}\right.$ we obtain

$$
\psi\left(\ell_{2}\right)-\psi\left(\ell_{1}\right)=\int_{\ell_{1}}^{\ell_{2}} \psi^{\prime}(\ell) d \ell=\int_{\psi^{\prime}\left(\ell_{2}\right)}^{\psi^{\prime}\left(\ell_{1}\right)} \psi^{\prime} \frac{L^{\prime \prime}\left(\psi^{\prime}\right)}{D L^{\prime}\left(\psi^{\prime}\right)} d \psi^{\prime}
$$

From the assumption of continuity of $L^{*}$, we have:

$$
\begin{gathered}
\lim _{\beta \rightarrow \infty} \int_{0}^{\beta} t L^{\prime \prime}(t) d t=\lim _{\beta \rightarrow \infty}\left(\left.t L^{\prime}(t)\right|_{0} ^{\beta}-\int_{0}^{\beta} L^{\prime \prime}(t) d t\right)=\lim _{\beta \rightarrow \infty} L^{*}\left(L^{\prime}(\beta)\right) \\
=\lim _{p \rightarrow \ell^{*}} L^{*}(p)=+\infty
\end{gathered}
$$

Applying Lemma 2, we obtain

$$
\lim _{\beta \rightarrow \infty} \int_{0}^{\beta} t \frac{L^{\prime \prime}(t)}{L^{\prime}(t)} d t=+\infty .
$$

Hence, for every $\alpha$, there exists $\beta(\alpha)$ such that

$$
\int_{\alpha}^{\beta(\alpha)} t \frac{L^{\prime \prime}(t)}{D L^{\prime}(t)} d t=v^{*}
$$

Fix $\tilde{\alpha}$ such that $\tilde{\alpha} d^{*} \geq v^{*}$. Let $\tilde{\psi}$ be the solution to equation (9) satisfying the initial conditions

$$
\tilde{\psi}(0)=0 ; \tilde{\psi}^{\prime}(0)=\beta(\tilde{\alpha})
$$


and define $d^{* *}$ setting $\tilde{\psi}^{\prime}\left(d^{* *}\right)=\tilde{\alpha}$. From equation (10) we obtain

$$
\tilde{\psi}\left(d^{* *}\right)=\int_{\psi^{\prime}\left(d^{* *}\right)}^{\psi^{\prime}(0)} \psi^{\prime} \frac{L^{\prime \prime}\left(\psi^{\prime}\right)}{D L^{\prime}\left(\psi^{\prime}\right)} d \psi^{\prime}=v^{*} .
$$

Call $\Omega_{i}^{* *}=\left\{x \in \Omega ; d\left(x, \partial \Omega_{i}\right) \leq d^{* *}\right\}$ and call $\partial_{i}^{* *}=\left\{x \in \Omega ; d\left(x, \partial \Omega_{i}\right)=d^{* *}\right\}$. We have obtained that, on $\partial_{i}^{* *}$, the map $\tilde{v}=k_{i}+\tilde{\psi}(d(x))$ is such that $\tilde{v}(x) \geq \tilde{w}(x)$. Moreover, $\tilde{\psi}^{\prime \prime}$, defined by $(9)$, is negative so that, on the interval $\left(0, d^{* *}\right), \tilde{\psi}^{\prime} \geq \tilde{\alpha}$. Hence, we have $v^{*}=\tilde{\psi}\left(d^{* *}\right) \geq \tilde{\alpha} d^{* *}$, while, from the choice of $\tilde{\alpha}, \tilde{\alpha} d^{*} \geq v^{*}$, so that $d^{*} \geq d^{* *}$. In addition, being $\tilde{\psi}^{\prime}>0$, from (9), we obtain

$$
\begin{gathered}
\operatorname{div}_{x} \nabla_{\xi} L(|\nabla \tilde{v}(x)|)=L^{\prime}\left(\left|\tilde{\psi}^{\prime}(d(x))\right|\right) \operatorname{sign}\left(\tilde{\psi}^{\prime}(d(x))\right) \Delta d(x)+\tilde{\psi}^{\prime \prime}(d(x)) L^{\prime \prime}\left(\left|\tilde{\psi}^{\prime}(d(x))\right|\right) \\
=L^{\prime}\left(\tilde{\psi}^{\prime}(d(x))\right) \Delta d(x)+\tilde{\psi}^{\prime \prime}(d(x)) L^{\prime \prime}\left(\tilde{\psi}^{\prime}(d(x))\right) \leq 0 .
\end{gathered}
$$

On $\left\{x \in \Omega: d(x)<d^{* *}\right\}, \tilde{v}$ is a Lipschitz continuous supersolution. In the case $\ell=\infty$, this proves the existence of a solution to the minimization problem (1) on $\phi+\operatorname{Lip}_{0}(\Omega)$.

ii) Case $\operatorname{Dom}(L)=(-\ell,+\ell)$. By the assumption of continuity, $\lim _{t \rightarrow \ell} L(t)=$ $+\infty$.

Let $\tilde{w}$ be a solution to problem $(1)$ on $\phi+W^{1,1}(\Omega)$ : such a solution exists and it is Lipschitz continuous.

a) We claim that there exits $\Lambda^{*}<\ell$ such that, when $d\left(x, \partial \Omega_{i}\right)=d^{*},\left|\tilde{w}(x)-k_{i}\right| \leq$ $\Lambda^{*} d^{*}$ : in fact, otherwise, let $\left(x_{n}\right)$ be such that $d\left(x_{n}\right)=d^{*}$ and $\left|\tilde{w}(x)-k_{i}\right| \rightarrow \ell d^{*} ; \mathrm{a}$ subsequence converges to $x^{*}$, and $\tilde{w}\left(x^{*}\right)-k_{i}=\ell d^{*}$, a contradiction to lemma 1 .

We consider $g$, as defined by (5), and we seek $r^{0}$ such that, setting

$$
\tilde{\psi}(r)=k_{i}+\int_{r^{*}}^{r} g\left(\frac{s}{r_{0}}\right) d s
$$

we have $\tilde{\psi}\left(r^{*}+d^{*}\right) \geq \Lambda^{*} d^{*}+k_{i}$, i.e., such that

$$
\frac{1}{d^{*}} \int_{r^{*}}^{r^{*}+d^{*}} \tilde{\psi}^{\prime}(s) d s=\frac{1}{d^{*}} \int_{r^{*}}^{r^{*}+d^{*}} g\left(\frac{s}{r^{0}}\right) d s \geq \Lambda^{*}
$$

It is enough to consider $\delta$ such that $t \leq \delta$ implies $g(t)>\Lambda^{*}$, and to choose $r^{0}$ so large that $\frac{r^{*}+d^{*}}{r^{0}}<\delta$ : with this choice, (11) holds.

To define a barrier near $\partial \Omega_{i}$, consider $v(d)=\tilde{\psi}\left(d+r^{*}\right)$ (so that $v^{\prime}(d)=\tilde{\psi}^{\prime}(d+$ $\left.r^{*}\right)=g\left(\frac{d+r^{*}}{r_{0}}\right), v^{\prime \prime}(d)=g^{\prime}\left(\frac{d+r^{*}}{r_{0}}\right) \frac{1}{r_{0}}$ and, in particular, $\left.v^{\prime}(d)>0\right)$ and set $\tilde{v}(x)=$ $v(d(x))$. On $\partial \Omega_{i}, \tilde{v}=v(0)=\tilde{\psi}\left(r^{*}\right)=k_{i}$ and, when $\operatorname{dist}\left(x, \partial \Omega_{i}\right)=d^{*}$, recalling (11) and the choice of $\Lambda^{*}$ in point a), we have $\tilde{v}(x)=\tilde{\psi}\left(r^{*}+d^{*}\right) \geq d^{*} \Lambda^{*}+k_{i} \geq \tilde{w}(x)$. Hence, to show that $\tilde{v}$ is an upper barrier, it is left to show that it is a supersolution, i.e., that, for $x \in \Omega$, we have $\operatorname{div}_{x} \nabla_{\xi} L(\|\nabla v(x)\|) \leq 0$.

Fix $x$, and recall that $\Delta d(x)=\sum_{1}^{N-1} \frac{1}{r_{i}(\pi(x))+d(x)}$. From $0<r^{*} \leq\left|r_{i}(\pi(x))\right|$ we obtain

$$
\sum_{1}^{N-1} \frac{1}{r_{i}(\pi(x))+d(x)} \leq \sum_{1}^{N-1} \frac{1}{\left|r_{i}(\pi(x))\right|+d(x)} \leq \frac{N-1}{r^{*}+d(x)}
$$

hence

$$
\begin{gathered}
\operatorname{div}_{x} \nabla_{\xi} L(|\nabla \tilde{v}(x)|)= \\
L^{\prime}\left(g\left(\frac{d(x)+r^{*}}{r_{0}}\right)\right) \sum_{1}^{N-1} \frac{1}{r_{i}(\pi(x))+d(x)}+\frac{1}{r_{0}} g^{\prime}\left(\frac{d(x)+r^{*}}{r_{0}}\right) L^{\prime \prime}\left(g\left(\frac{d(x)+r^{*}}{r_{0}}\right)\right)
\end{gathered}
$$




$$
\leq \frac{1}{r_{0}}\left[L^{\prime}\left(g\left(\frac{d(x)+r^{*}}{r_{0}}\right)\right) \frac{r_{0}(N-1)}{r^{*}+d(x)}+g^{\prime}\left(\frac{d(x)+r^{*}}{r_{0}}\right) L^{\prime \prime}\left(g\left(\frac{d(x)+r^{*}}{r_{0}}\right)\right)\right]=0,
$$

since $g$ satisfies $(6)$.

The above ends the proof that $\tilde{v}$ is a barrier, thus showing the existence of a solution on $\phi+\operatorname{Lip}_{0}(\Omega)[2]$.

iii) Points i) and ii) above prove the existence of a solution $\tilde{w}$ in the class of Lipschitz continuous functions. We wish to show that $\tilde{w}$ is a solution in $\phi+W_{0}^{1,1}(\Omega)$. By Proposition 2, the Euler Lagrange equation holds. From the convexity of $L$, one obtains that

$$
\int_{\Omega} L(|\nabla \tilde{w}(x)+\nabla \eta(x)|) d x \geq \int_{\Omega} L(|\nabla \tilde{w}(x)|) d x+\int_{\Omega}\langle\nabla L(|\nabla \tilde{w}(x)|), \nabla \eta(x)\rangle d x
$$

thus proving that $\tilde{w}$ is a solution in $\phi+W_{0}^{1,1}(\Omega)$.

\section{REFERENCES}

[1] Gilbarg, D; Trudinger, N. S. Elliptic partial differential equations of second order. Reprint of the 1998 edition. Classics in Mathematics. Springer-Verlag, Berlin, 2001.

[2] Giusti, E. Minimal surfaces and functions of bounded variation. Monographs in Mathematics, 80. Birkhuser Verlag, Basel, 1984.

[3] Jenkins, H.; Serrin, J. The Dirichlet problem for the minimal surface equation in higher dimensions. J. Reine Angew. Math. 2291968 170-187.

[4] Jenkins, H.; Serrin, J. Variational problems of minimal surface type. II. Boundary value problems for the minimal surface equation. Arch. Rational Mech. Anal. 211966 321-342.

[5] Hartman, Ph.; Stampacchia, G. On some non-linear elliptic differential-functional equations. Acta Math. 115 (1966), 271-310.

[6] Rockafellar, R. T. , Convex Analysis, Princeton University Press, Princeton, NJ, 1972.

Acknowledgment. The authors are indebted to an unknown referee for the kind and helpful remarks.

Dipartimento di Matematica e Applicazioni, Università degli Studi di Milano-Bicocca, Via R. Cozzi 53, 20125 Milano, Italy

Departamento de Matemática Rua Romão Ramalho, 59 7000-671 Évora, Portugal 